

ECOTOX - BRASIL

\title{
Avaliação da Toxicidade Aguda de Piraclostrobin, Epoxiconazol e sua Mistura em Colossoma macropomum (Tambaqui)
}

\author{
E.B. Prestes; Z. Clemente; V.L.S.S. Castro; C.M. Jonsson \\ Laboratório de Ecotoxicologia e Biossegurança, Embrapa Meio Ambiente, Jaguariúna, SP, Brasil.
}

(Received May 20, 2012; Accept April 28, 2013)

\begin{abstract}
Resumo
O presente trabalho avaliou o efeito toxicológico de formulações fungicidas à base de piraclostrobin e epoxiconazol, isoladamente e em formulação conjugada sobre Colossoma macropomum (tambaqui) por 96 horas através da determinação da Concentração Letal Média $\left(\mathrm{CL}_{50-96 \mathrm{~h}}\right)$ de cada uma das formulações. Foram encontrados valores de $\mathrm{CL}_{50-96 \mathrm{~h}}$ equivalentes a $0,018 \mathrm{mg} \mathrm{L}^{-1}$ para o piraclostrobin, $2,28 \mathrm{mg} \mathrm{L}^{-1}$ para o epoxiconazol e $0,021 \mathrm{mg} \mathrm{L}^{-1}$ para sua formulação conjugada. Das três formulações utilizadas, o piraclostrobin isoladamente mostrou-se o mais tóxico para os organismos estudados e o uso conjugado das duas substâncias nas concentrações estudadas apresentou um fraco efeito sinérgico.
\end{abstract}

Palavras-chave: Colossoma macropomum, epoxiconazol, piraclostrobin, toxicidade.

Acute Toxicity Evalution of Pyraclostrobin, Epoxiconazole and

a Combined Formulation to Colossoma macropomum (tambaqui)

\begin{abstract}
The current paper investigates toxicological effects of fungicidal formulations based on pyraclostrobin, epoxiconazole and a combined formulation to Colossoma macropomum (tambaqui) during 96 hours by determination of Mean Lethal Concentration $\left(\mathrm{LC}_{50-96 \mathrm{~h}}\right.$ ) of each formulation. $\mathrm{LC}_{50-99 \mathrm{~h}}$ values found were $0.018 \mathrm{mg} \mathrm{L}^{-1}, 2.28 \mathrm{mg} \mathrm{L}^{-1}$ and $0.021 \mathrm{mg} \mathrm{L}^{-1}$ for pyraclostrobin, epoxiconazole and combined formulation, respectively. Pyraclostrobin isolatedly was the most toxic chemical among analyzed formulations and the combined use of those two fungicides presented a weak synergic effect, regarding the concentration tested.
\end{abstract}

Key words: Colossoma macropomum, epoxiconazole, pyraclostrobin, toxicity.

\section{INTRODUÇÃO}

O uso combinado de dois ou mais princípios ativos fungicidas na agricultura vem sendo recomendado como forma de incrementar a sinergia entre os diferentes agentes utilizados e retardando a ocorrência de resistência fúngica (Gisi, 1996). No mercado brasileiro são encontradas várias formulações combinadas de fungicidas e entre os quais, os pertencentes às classes dos triazóis e estrobilurinas, como o epoxiconazol e o piraclostrobin.
A despeito de seu papel como ferramenta para o aumento da produtividade dos cultivos, os fungicidas podem promover a contaminação da água e solos das áreas de cultivo e adjacências, comprometendo a fauna e flora presentes (Battaglin et al., 2011; Silva et al., 2009). Apesar de serem encontrados na literatura trabalhos relativos aos efeitos ecotoxicológicos dos triazóis sobre organismos aquáticos, são poucos os trabalhos sobre as estrobilurinas avaliadas individualmente e em especial sobre seu uso quando combinadas em um único produto. 
Deve ser observado, ainda, que para a liberação de um fungicida ao mercado, as agências reguladoras consideram a toxicidade dos compostos químicos sobre organismos não-alvo de maneira individualizada, desconsiderando que no ambiente natural a toxicidade ocorre através da mistura de diversos contaminantes que podem interagir sob diversas formas, inclusive com o aumento de toxicidade através de interações sinérgicas.

A ação dos triazóis decorre da inibição do esterol $14 \alpha$-demetilase (codificado pelo gene CYP51) em bolores e leveduras, bloqueando a biossíntese do ergosterol, componente essencial das membranas celulares destes organismos (Zarn et al., 2003). Já as estrobilurinas agem inibindo a respiração mitocondrial pela ligação ao citocromo $b$, bloqueando assim a geração de ATP (Bartlett et al., 2001).

Dessa forma, foi determinada a Concentração Letal Média $\left(\mathrm{CL}_{50-96 h}\right)$ de fungicidas a base de piraclostrobin, epoxiconazol e sua mistura, como forma de avaliar a toxicidade aguda desses produtos em peixes de origem brasileira da espécie Colossoma macropomum (tambaqui).

\section{MATERIAL E MÉTODOS}

Foram testadas formulações de fungicidas à base de piraclostrobin $(25,0 \% \mathrm{~m} / \mathrm{v})$, epoxiconazol $(12,5 \% \mathrm{~m} / \mathrm{v})$ e de sua mistura (piraclostrobin a $13,3 \% \mathrm{~m} / \mathrm{v}$ e epoxiconazol a $5,0 \% \mathrm{~m} / \mathrm{v}$ ), oriundas de apresentações comerciais disponíveis no mercado. Como organismo-teste utilizou-se o peixe Colossoma macropomum (tambaqui) com peso médio de 4 gramas. Os alevinos foram submetidos à aclimatação doze dias antes da exposição aos agentes fungicidas em tanques com água declorinizada com dureza total de $36 \mathrm{mg} \mathrm{L}^{-1}$ de $\mathrm{CaCO}_{3}, \mathrm{pH} 7,2$, temperatura de $27 \pm 2{ }^{\circ} \mathrm{C}$, fotoperíodo de 12 horas e alimentados uma vez ao dia. Tais condições ambientais foram mantidas durante todo o experimento, à exceção da alimentação, que foi suspensa a partir do primeiro dia de exposição aos fungicidas. Sete dias antes da exposição aos agentes, foram formados grupos com 5 animais cada, sendo estes alocados em aquários observando-se a proporção de $1 \mathrm{~L}$ de água por grama de peixe. A exposição dos animais ocorreu pelo período de 96 horas, utilizando-se do piraclostrobin nas concentrações de 0,$009 ; 0,020 ; 0 ; 030$; 0,050 e $0,100 \mathrm{mg} \mathrm{L}^{-1}$; do epoxiconazol nas concentrações de 0,$950 ; 1,700 ; 3,100 ; 5,500$ e $10,000 \mathrm{mg} \mathrm{L}^{-1}$ e da mistura dos fungicidas piraclostrobin $(13,3 \% \mathrm{~m} / \mathrm{v})$ e epoxiconazol $(5 \% \mathrm{~m} / \mathrm{v})$ nas concentrações de 0,$009 ; 0,020 ; 0,030 ; 0,050$ e $1,000 \mathrm{mg} \mathrm{L}^{-1}$, além da manutenção de grupos-controle. Para cada uma das concentrações testadas foram expostos dois grupos de peixes. A definição das concentrações utilizadas baseou-se em estudos prévios de toxicidade aguda pelo grupo de trabalho.

A mortalidade de cada grupo foi determinada às 24,48 , 72 e 96 horas de exposição, servindo de base para o cálculo da Concentração Letal Média $\mathrm{CL}_{50-96 \mathrm{~h}}$ e de seus respectivos intervalos de confiança a 95\% utilizando-se o programa Statgraphics Plus (Manugistics, 2001). A análise isobolográfica e o cálculo do Índice de Aditividade foram realizados conforme descritos por Marking (1977, 1985), permitindo avaliar a ocorrência de efeito sinérgico, antagônico ou aditivo dos ingredientes na mistura sobre o organismo-teste.

\section{RESULTADOS E DISCUSSÃO}

Os testes de toxicidade aguda em fungicidas permitem uma avaliação rápida sobre o impacto destes agentes sobre organismos expostos aos mesmos, permitindo estimar seus efeitos imediatos sobre a fauna após sua utilização no ambiente. Estes estudos servem ainda de base para o desenho dos testes de toxicidade crônica, estes importantes para a estimação dos efeitos de quantidades residuais dos agentes por um período prolongado. A obtenção de dados através dos diferentes testes de toxicidade se torna relevante à medida que novos agentes fungicidas são lançados no mercado e poucos são os trabalhos que avaliam os seus efeitos em grupos representativos dos diferentes níveis tróficos.

No presente trabalho o piraclostrobin foi o fungicida mais tóxico aos peixes dentre os agentes testados, apresentando uma $\mathrm{CL}_{50-96 \mathrm{~h}}$ equivalente a $0,018 \mathrm{mg} \mathrm{L}^{-1}$, sendo seguido pela mistura piraclostrobin + epoxiconazol e por último pelo epoxiconazol (Tabela 1).

Associando os resultados do presente trabalho e a classificação internacional para riscos agudos ao ambiente aquático (Globally Harmonized System of Classification and Labelling of Chemicals - GHS, United Nations, 2009) o piraclostrobin e a formulação combinada de piraclostrobin + epoxiconazol enquadram-se como compostos de Categoria 1 - "muito tóxico à vida aquática", enquanto o epoxiconazol como fungicida isolado classifica-se na Categoria 2 - "tóxico à vida aquática".

A agência reguladora de pesticidas da Austrália (APVMA, 2003) registra a $\mathrm{CL}_{50}$ de piraclostrobin próxima aos valores determinados neste trabalho, variando de $0,019-0,034 \mathrm{mg}$ $\mathrm{L}^{-1}$ para a perca-sol (Lepomis macrochirus) e 0,012-0,026 $\mathrm{mg} \mathrm{L} \mathrm{L}^{-1}$ para a carpa (Cyprinus carpio). Valores próximos são disponibilizados pela agência de segurança alimentar européia (EFSA, 2008), que informa, após testes com diversas espécies de peixes, $\mathrm{CL}_{50}$ de epoxiconazol entre 3,14 - 8,27 mg L $\mathrm{m}^{-1}$, sendo a truta (Oncorrynchus sp.) a espécie mais sensível. Por outro lado, Kreutz et al. (2008) expondo

Tabela 1 - Concentração Letal Média $\left(\mathrm{CL}_{50-96 h}\right)$ e equação de regressão da dispersão de mortalidade de Colossoma macropomum submetidos a diferentes concentrações de piraclostrobin, epoxiconazol e a mistura de piraclostrobin $(13,3 \%)+$ epoxiconazol $(5,0 \%)$ por 96 horas.

\begin{tabular}{lccc}
\hline Fungicida & $\mathbf{C L}_{\mathbf{5 0 - 9 6} \mathbf{h}}$ & Equação de regressão & ${ }^{*} \mathbf{R}^{\mathbf{2}}$ \\
\hline Piraclostrobin & $0,018 \mathrm{mg} \mathrm{L}^{-1}$ & $\mathrm{y}=-11,198+623,993 \mathrm{x}$ & 0,89 \\
$\begin{array}{l}\text { Epoxiconazol } \\
\text { Mistura }\end{array}$ & $2,280 \mathrm{mg} \mathrm{L}^{-1}$ & $\mathrm{y}=-3,755+1,644 \mathrm{x}$ & 0,82 \\
$\begin{array}{l}\text { (piraclostrobin }+ \\
\text { epoxiconazol) }\end{array}$ & $0,021 \mathrm{mg} \mathrm{L}^{-1}$ & $\mathrm{y}=-12,675+607,533 \mathrm{x}$ & 0,78 \\
\hline
\end{tabular}

$* \mathrm{R}^{2}=$ coeficiente de determinação 
jundiás (Rhamdia quelen) a formulação combinada idêntica de fungicidas (piraclostrobin + epoxiconazol) determinou um valor de $9,9 \mathrm{mg} \mathrm{L}^{-1}$ para $\mathrm{CL}_{50-99 \mathrm{~h}}$, o que foi muito acima do encontrado neste experimento.

A variabilidade na sensibilidade entre diferentes espécies é um fator a ser ponderado quando da execução de provas em animais. Neste ínterim, Breuer (2004) em estudo de toxicidade aguda com outra estrobilurina (fluoxastrobin), expôs trutas e percas-sóis ao fungicida, determinando valores de $\mathrm{CL}_{50-96 \mathrm{~h}}$ que variaram de 0,33 $\mathrm{mg} \mathrm{L}^{-1}$ a $0,97 \mathrm{~m} \mathrm{~L}^{-1}$, para cada uma das espécies.

A análise isobolográfica (Figura 1) e o cálculo do Índice de Aditividade (IA) equivalente a 0,17 sugerem que o efeito da combinação entre os dois fungicidas na proporção utilizada apresenta pouco efeito sinérgico, sendo a formulação associada de piraclostrobin e epoxiconazol testada apenas 1,17 vezes mais tóxica que os fungicidas em separado, em relação à toxicidade aguda sobre o Colossoma macropoтит.

Todavia, deve-se considerar que essa sinergia pode ser relevante para outros organismos aquáticos não-alvo. Neste contexto, o trabalho de Prestes et al. (2011) relata que a combinação semelhante de fungicidas exerce um forte efeito sinérgico sobre algas da espécie Pseudokirchneriella subcapitata, com fator de magnificação de toxicidade de 13,6 vezes em relação à toxicidade individual de cada composto. A variação da sensibilidade entre espécies não-alvo fica evidente quando consideramos os mesmos fungicidas utilizados neste trabalho testados em indivíduos de outros níveis tróficos em seus respectivos testes de toxicidade aguda (Tabela 2).

Os resultados do presente trabalho são úteis no planejamento de estudos de longo prazo para a comparação de toxicidade entre fungicidas, em especial para os agentes estudados, além do fornecimento de dados sobre interações de poluentes em organismos não-alvo. Neste ínterim, o trabalho traz ainda a possibilidade da utilização do tambaqui como alternativa interessante para testes de toxicidade, dada a disponibilidade, custo de aquisição e facilidade de manutenção desta espécie sob condições de laboratório.

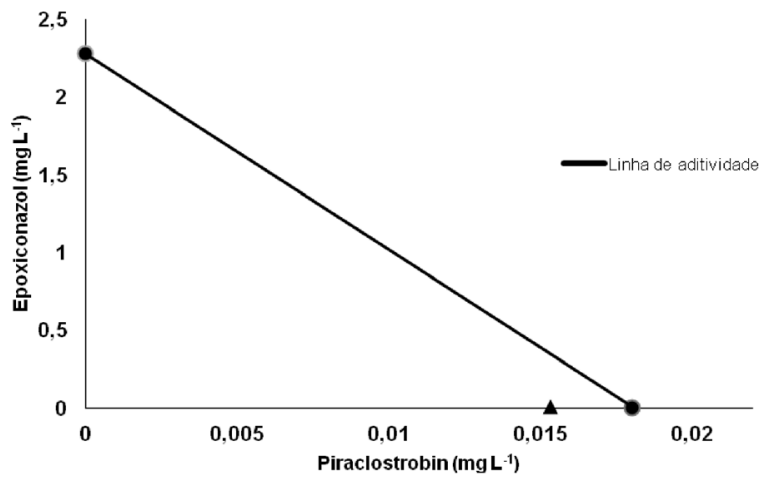

Figura 1 - Análise isobolográfica da toxicidade de piraclostrobin, epoxiconazol e sua mistura em peixes. O ponto triangular abaixo da reta representa a concentração da combinação piraclostrobin + epoxiconazol que causa $50 \%$ de morte de um grupo de peixes expostos. O Índice de Aditividade (IA) calculado para este ponto equivale a 0,17 .
Tabela 2 - Concentração Letal Média $\left(\mathrm{CL}_{50}\right)$ de espécies representantes de três diferentes níveis tróficos submetidos a diferentes concentrações de piraclostrobin, epoxiconazol e a mistura de piraclostrobin $(13,3 \%)+$ epoxiconazol $(5,0 \%)$.

\begin{tabular}{lccc}
\hline Fungicida & $\begin{array}{c}\text { Alga } \\
\boldsymbol{P}_{\text {subcapitata }} \mathbf{~}^{\left(\mathbf{C L}_{50-72 \mathrm{~h}}\right)}\end{array}$ & $\begin{array}{c}\text { Microcrustáceo } \\
\text { D. similis }^{2} \\
\left(\mathbf{C L}_{50-48 \mathrm{~h}}\right)\end{array}$ & $\begin{array}{c}\text { Peixe } \\
\text { C. } \text { macropomum }^{3} \\
\left(\mathbf{C L}_{50-96 \mathrm{~h}}\right)\end{array}$ \\
\hline Piraclostrobin & $5,57 \mathrm{mg} \mathrm{L}^{-1}$ & $0,018 \mathrm{mg} \mathrm{L}^{-1}$ & $0,018 \mathrm{mg} \mathrm{L}^{-1}$ \\
Epoxiconazol & $1,14 \mathrm{mg} \mathrm{L}^{-1}$ & $0,090 \mathrm{mg} \mathrm{L}^{-1}$ & $2,280 \mathrm{mg} \mathrm{L}^{-1}$ \\
$\begin{array}{l}\text { Mistura } \\
\text { (piraclostrobin }+ \\
\text { epoxiconazol) }\end{array}$ & $0,20 \mathrm{mg} \mathrm{L}^{-1}$ & $0,023 \mathrm{mg} \mathrm{L}^{-1}$ & $0,021 \mathrm{mg} \mathrm{L}^{-1}$ \\
\hline
\end{tabular}

Fontes: 1 - Prestes et al. (2011); 2 - Prestes et al. (2010); 3 - resultados deste trabalho.

\section{AGRADECIMENTOS}

Os autores agradecem ao Conselho Nacional de Desenvolvimento Científico e Tecnológico (CNPq) e à Fundação de Amparo à Pesquisa do Estado de São Paulo (FAPESP) pelo suporte financeiro ao estudo.

\section{REFERÊNCIAS}

APVMA - AUSTRALIAN PESTICIDES AND VETERINARY MEDICINES AUTHORITY, 2003, Evaluation of the new active Pyraclostrobin in the product Cabrio fungicide. Canberra: APVMA. 49p.

BARTletT, D. W., ClOUGH, J. M., GODFrey, C. R. A., GODWIN, J. R., HALL, A. A., HEANEY, S. P. \& MAUND, S. J., 2001, Understanding the strobilurin fungicides. Pestic. Outlook, 4: 143-148. doi: 10.1039/b106300f

BATTAGLin, A., W., SANDSTROM, M. W., KUIVILA, K. M., KOLPIN, D. N. \& MEYER, M. T., 2011, Occurrence of azoxystrobin, propiconazole and selected other fungicides in US streams, 2005-2006. Water, Air, Soil Pollut., 218: 307-322. doi: 10.1007/s11270-010-0643-2

BREUER, P., 2004, Ecotoxicological profile of the fungicide Fluoxastrobin. Pflanzenschutz-Nachr. Bayer, 57: 319-336.

EFSA - EUROPEAN FOOD SAFETY AGENCY, 2008, Conclusion regarding the peer review of the pesticide risk assessment of the active substance epoxiconazole. EFSA Scientific Report, 138: 1-80. doi:10.2903/j.efsa.2008.138r

GISI, U. 1996, Synergistic interaction of fungicides in mixtures. Phytopathology, 86: 1273-1279.

KREUTZ, L. C., BARCELLOS, L. J. G., SILVA, T. O., ANZILIERO, D., MARTINS, D., LORENSON, M. MARTENINGHE, A. \& SILVA, L. B., 2008, Acute toxicity test of agricultural pesticides on silver catfish (Rhamdia quelen) fingerlings. Cienc. Rural, 38: 1050-1055. doi: 10.1590/S0103-84782008000400022

MANUGISTICS, 2001, Statgraphics Plus. Version 5.1 for Windows. Rockville: Manugistics Group.

MARKING, L. L., 1977, Method for assessing additive toxicity of chemical mixtures, pp. 99-108. In: F. L. MAYER \& J. L. HAMELINK (orgs.), Aquatic Toxicology and Hazard Evaluation. Philadelphia: American Society for Testing and Materials.

MARKING, L. L., 1985, Toxicity of chemical mixtures, pp. 164-176. In: G. M. RAND \& S. R. PETROCELLI (orgs.), Fundamentals 
of Aquatic Toxicology: methods and applications. Washington: Hemisphere.

PRESTES, E. B., JONSSON, C. M. \& CASTRO, V. L. S. S. DE, 2011, Avaliação da toxicidade de piraclostrobin, epoxiconazol e sua mistura em alga Pseudokirchneriella subcapitata. Pesticidas: R. Ecotoxicol. e Meio Ambiente, 21: 39-46.

PRESTES, E. B., PAULA, V. J. DE, SANTOS, F., JONSSON, C. M. \& CASTRO, V. L., 2010, Estudo sobre a ação conjunta dos fungicidas epoxiconazol e piraclostrobina na mobilidade do microcrustáceo Daphnia similis. In: $11^{\circ}$ Congresso Brasileiro de Ecotoxicologia, Bombinhas, Santa Catarina.
SILVA, D. R. O., AVILA, L. A., AGOSTINETTO, D. \& DAL MAGRO, T., 2009, Monitoramento de agrotóxicos em águas superficiais de regiões orizícolas no sul do Brasil. Cienc. Rural, 39: 2383-2389. doi: 10.1590/S0103-84782009000900001

UNITED NATIONS, 2009, Globally harmonized system of classification and labeling of chemicals (GHS). 3 Ed. New York and Geneva: United Nations. 566p.

ZARN, A. J., BRÜSCHWEILER, B. J. \& SCHLATTER, J. R., 2003, Azole fungicides affect mammalian steroidogenesis by inhibiting sterol 14 alpha-demethylase and aromatase. Environ. Health Perspect. 111: 255-261. 